\title{
The use of gold markers and electronic portal imaging for radiotherapy verification in prostate cancer patients: Sweden Ghana Medical Centre experience
}

\author{
George Felix Acquah \\ Department of Radiation Oncology Physics, Sweden Ghana Medical Centre, East Legon, Ghana.
}

Received January 09, 2014; Revised February 03, 2014; Accepted February 08, 2014; Published Online February 11, 2014

\section{Educational Report}

\begin{abstract}
The success of radiotherapy cancer treatment delivery depends on the accuracy of patient setup for each fraction. A significant problem arises from reproducing the same patient position and prostate location during treatment planning for every fraction of the treatment process. To analyze the daily movements of the prostate, gold markers are implanted in the prostate and portal images taken and manually matched with reference images to locate the prostate. Geometrical and fiducial markers are annotated onto a highly quality generated digitally reconstructed radiographs, that are compared with portal images acquired right before treatment dose delivery. A 0 and 270 degree treatment fields are used to calculate prostate shifts for all prostate cancer patients undergoing treatment at the Sweden Ghana Medical Centre, using an iViewGT portal imaging device. After aligning of the marker positions onto the reference images, the set-up deviations corrections are displayed and an on-line correction procedure applied. The measured migrations of the prostate markers are below the threshold of $3 \mathrm{~mm}$ for the main plans and $2 \mathrm{~mm}$ for the boost plans. With daily electronic portal imaging combined with gold markers, provides an objective method for verifying and correcting the position of the prostate immediately prior to radiation delivery.
\end{abstract}

Keywords: Prostate Cancer; Gold Markers; Treatment Verification; EPID; DRRs

\section{Introduction}

External beam radiation therapy is an effective method of treating cancer, in which the lesion is irradiated with high-energy beams produced by a linear accelerator. The goal is to minimize the dose to normal tissue surrounding the clinical target volume (CTV). Treatment techniques such as three dimensional conformal radiotherapy (3DCRT) and intensity modulated radiation therapy (IMRT) provide very accurate radiation to the lesion, while sparing healthy tissues. Radiation treatment always starts with the acquisition of planning computerized tomographic (CT) images. CT data play a central role in radiotherapy treatment planning (RTP) ${ }^{1}$ Efficacy of the RTP depends on the patient setup at each daily fraction. The problem is to reproduce the patient position at the time of acquiring the planning CT scans (used for RTP)

Corresponding author: George Felix Acquah; Sweden Ghana Medical Centre, Radiation Oncology Medical Physics Department, Cantonments, Accra, Ghana. Email: felixfelyx@yahoo.com

Cite this article as:

Acquah GF. The use of gold markers and electronic portal imaging for radiotherapy verification in prostate cancer patients: Sweden Ghana Medical Centre experience. Int J Cancer Ther Oncol 2014; 2(1):020112. DOI: 10.14319/ijcto.0201.12 for each fraction of the treatment process. Discrepancies between the planned and delivered treatment positions significantly degrade the therapeutic ratio. The planning target volume (PTV) is defined to include the CTV and associated treatment uncertainties, which include but are not limited to imaging, patient setup, and organ motion. Prostate motion, defined as a positional change of the prostate at the time of treatment relative to the planning position, has been evaluated for different conditions and methods. Radiation oncologists are now required to be familiar with 3-dimensional determination of gross tumour volume (GTV), clinical target volume, and organs at risk. Setup error in CT simulation also needs to be paid more attention, as recent studies pointed out. ${ }^{2}$ However, it could be difficult for an oncologist to meet these increasing requirements; hence, distant consultation is expected to be useful in the management of patients. Sending images needed for radiotherapy seems to be one means of such consultation. Radio opaque markers, gold seeds, CT-CT fusion, and CT chamfer matching are examples of methods used to determine the prostate position relative to fixed bony landmarks. The largest motion was observed to be in the anterior/posterior (AP) direction and to a lesser extent in the 
superior/inferior (SI) direction. The AP movement was strongly correlated with rectal filling and then to a lesser extent with bladder filling. ${ }^{3}$ One of the conventional methods to reduce patient setup error includes matching the skin tattoo marks generated at the planning phase with the treatment room laser lights. Immobilization devices such as foam casts or thermoplastic masks are also used to assist in reproducing the daily treatment position. These approaches can fail to adequately ensure the location reproducibility, since the positioning is done based on the outer surface of the patient's body. ${ }^{4}$ Adjustment of the patient setup can also be achieved using internal anatomical landmarks identified on portal images acquired with the patient in the treatment position. Portal images can be acquired using an electronic portal imaging device (EPID). ${ }^{1}$ Improper knowledge of the patient's anatomy and position during the course of therapy has always been a major source of concern in radiation therapy potentially compromising the clinical results by insufficient dose coverage of the target volume and/or over dosage to normal tissues. The management of target localization emanates in the concept of treatment margins, such as those described in the ICRU reports 50 and 62.5, 6

A critical requirement in radiation therapy is accurate day-to-day treatment setup. Early studies based on port films indicated the benefits of portal verification. ${ }^{7}$ Precise imaging and targeting of treatment targets for radiotherapy allows dose escalation while avoiding toxicity of the surrounding normal tissues. The aim of imaging for treatment verification is to evaluate the geometric uncertainties both due to organ motion and setup variations. Portal imaging using bony structures can only detect set-up variations. If implanted markers are used, both set-up variations and internal organ motion can be detected and if necessary, corrected. Two types of deviations have to be distinguished: the day-to-day (random) variations and the systematic variations, present during all treatment fractions. ${ }^{8}$ Both the set-up variations and the organ motion have random and systematic components. The use of gold markers for positioning verification has been reported mainly for the irradiation of the prostate; investigations have started for head-and-neck, lung and liver. ${ }^{1}$

In prostate radiotherapy ${ }^{3,9,10}$, the actual position of the prostate can be visualized and verified by using gold markers. In Sweden Ghana Medical centre (SGMC), so far, fiducial gold markers are implanted in prostate cancer patients who received external radiation therapy with $3 \mathrm{D}$ conformal radiotherapy planning technique for treatment. Three fine gold markers are inserted in the prostate under trans-rectal ultrasound guidance by an urologist. To verify the treatment position of the prostate using an iViewGT verification system, portal images are acquired with an electronic portal imaging device ${ }^{4,11}$, using the first 5 monitor units (MUs); 3MUs from the lateral and $2 \mathrm{MUs}$ from posterior of the treatment beams. The full standard configuration of an iViewGT portal imaging device includes a flat panel image detection system with powerful image processing and display, using industry standard IT hardware and software. To analyse patient and prostate position variations, the marker contours derived from the digitally reconstructed radiograph (DRR), are displayed overlaying the portal image and manually matched as shown in Figure 1.

In radiotherapy, a digitally reconstructed radiograph, which is used for treatment verification in CT simulation, is one of the important images which can be sent via telecommunication. Accurate and routine target localization is necessary for successful outcome in radiation therapy treatments. Electronic portal imaging devices provide an advanced tool with digital technology to improve target localization and maintain clinical efficiency. EPIDs are ubiquitous in the radiation therapy clinic, and they provide a powerful and flexible tool to collect and process data in a quantitative manner to improve treatment accuracy for virtually any treatment site. ${ }^{12}$
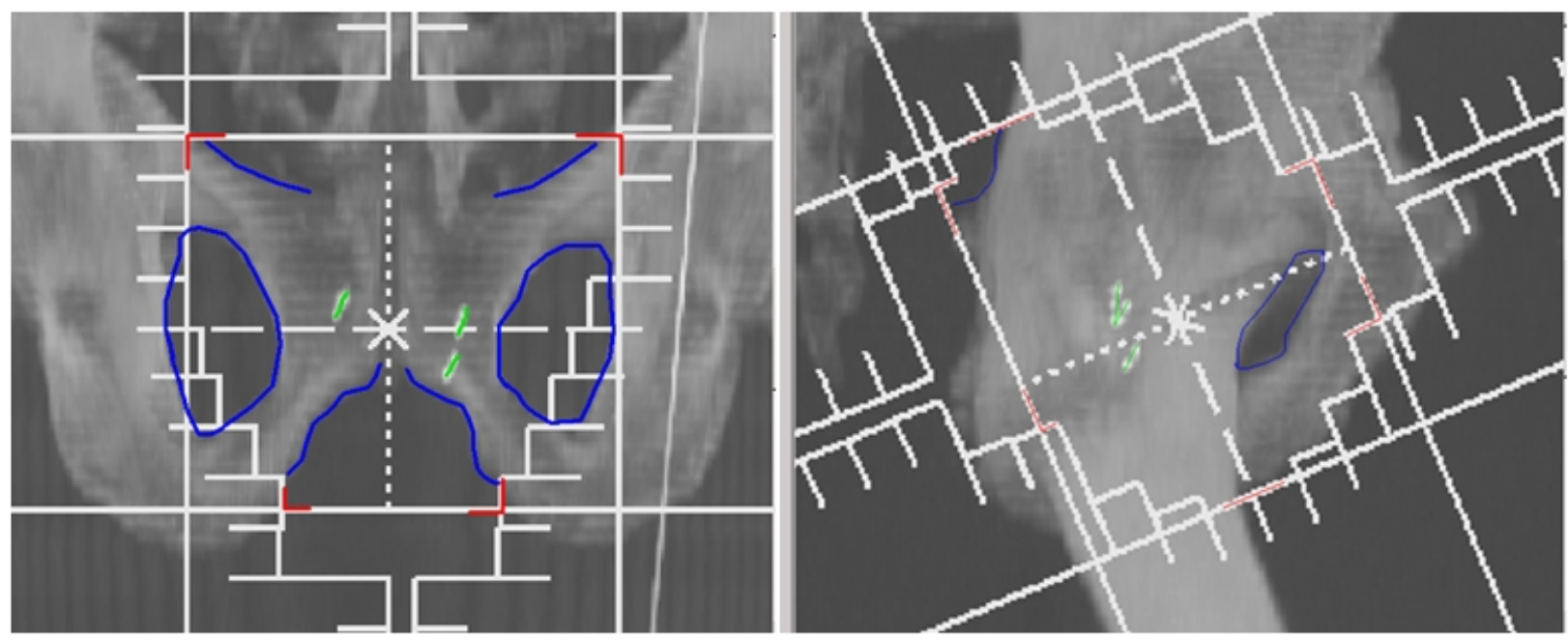

FIG. 1: Gold markers (green) and bone structure (blue) annotations with the treatment fields. 
Radiation therapy can only be effective if the intended dose is delivered as prescribed throughout the treatment course. Taking the impact of each of these variations into account for target localization is essential in providing the most accurate radiation treatment. Although many mechanisms exist to monitor and manage target positioning, the application of electronic portal imaging devices is becoming a standard tool for this purpose and is the focus of this article. ${ }^{13}$ At the planning stage a set of DRRs is generated from the CT scan of the patient. This set of images is used as the reference, representing the ideal patient position at the time of treatment. At the treatment stage a set of localization portal images is taken at the same gantry angles as the reference images. Localization and reference images are visually compared and corresponding anatomical landmarks are identified in both sets. The setup error is estimated and the patient position is corrected to reduce the discrepancies between the localization and reference images.

From the results, the random error in the prostate position, derived from the gold markers, is defined as the standard deviation of the day-to-day variations, averaged over all patients in the group. The systematic error is defined as the standard deviation of the distribution of average prostate deviations per patient. For inter-fraction prostate position corrections, the so-called No Action Level (NAL) off-line protocol can be used. With this protocol the systematic errors can be reduced, with a minimum of extra workload and hardly any extra treatment time necessary on the accelerator. The deviations are measured and corrected after the third treatment fraction. To correct both systematic and random errors, an on-line correction procedure is applied. ${ }^{14}$ Set-up deviations in lateral, longitudinal and vertical directions are corrected and checked again, before the irradiation.

Overall, the measured migrations of the prostate markers are expected to be in a threshold of $3 \mathrm{~mm}$ for the main plan and $2 \mathrm{~mm}$ for the boost plan. The results (in $\mathrm{mm}$ ) are prostate position relative to isocenter systematic deviation and the shifts are isocenter shifts not couch shifts. Implanted fiducial markers allow detection and correction of organ and/or tumour specific position variations. This image guided radiotherapy procedure with gold markers and the EPID has resulted in accurate high-dose, high-precision prostate treatments, with less toxicity to the bladder and rectum. Depending on accuracy requirements, off-line correction protocols appear to be effective in reducing systematic deviations at an acceptable workload. With on-line corrections a very high geometric accuracy $(<3 \mathrm{~mm})$ can be obtained, but at the cost of a considerably larger effort.

\section{Conclusion}

Prostate is known to move independently of bony landmarks requiring additional effort to visualize its position prior to radiation delivery. The use of implanted markers and electronic portal imaging has become the gold standard for daily verification of position and correction of patient setup and organ displacement errors. ${ }^{15}$ Improving geometric accuracy through image guided therapy in radiation treatment delivery can reduce the volume of normal tissue irradiated. There are various analysing methods to quantitatively assess the geometric gains from IGRT. One application of IGRT at SGMC involves implanting gold markers in prostate cancer patient irradiation setup. An EPID imaging method is used to bring the treatment fields into alignment with a reference DRR images. This method assumes a high degree of correlation between the prostate target and reference target location in real time. This reduction in errors due to target motion permits the use of high precise doses to the target, which in turn reduce dose to normal tissues. The gain in IGRT is realized by acquiring port film setup images on a daily basis to ensure appropriate field placement. Daily electronic portal imaging combined with gold markers provides an objective method to verify and correct the position of the prostate immediately prior to radiation delivery. Its routine clinical use can improve the precision of external beam radiation therapy in the treatment of prostate cancer. ${ }^{16,17} \mathrm{~A}$ total of 5 monitor units are used for imaging of these open isofields daily or 5 monitor units subtracted from the treatment fields.

\section{Conflict of interest}

The authors declare that they have no conflicts of interest. The authors alone are responsible for the content and writing of the paper.

\section{References}

1. Herman MG, Balter JM, Jaffray DA, et al. Clinical use of electronic portal imaging: report of AAPM Radiation Therapy Committee Task Group 58. Med Phys 2001; 28:713-34.

2. McKenzie AL, van Herk M, Mijnheer B. The width of margins in radiotherapy treatment plans. Phys Med Biol 2000; 45:3331- 42.

3. Hanley J, Lumley MA, Mageras GS, et al. Measurement of patient positioning errors in three-dimensional conformal radiotherapy of the prostate. Int J Radiat Oncol Biol Phys 1997; 37: 435-44.

4. Mageras GS. Management of target localization uncertainties in external-beam therapy. Semin Radiat Oncol 2005; 15:133-35.

5. International Commission on Radiation Units and Measurements (ICRU). Prescribing, Recording and reporting photon beam therapy, ICRU report 50). Bethesda, Maryland: ICRU Publications; 1993: 1-71.

6. International Commission on Radiation Units and Measurements (ICRU). Prescribing, Recording and 
reporting photon beam therapy (supplement to ICRU report 50), ICRU Report 62. Bethesda, Maryland: ICRU Publications; 1999: 1-52.

7. Balter JM, Sandler HM, Lam K, Bree RL, Lichter AS, ten Haken RK. Measurement of prostate movement over the course of routine radiotherapy using implanted markers. Int J Radiat Oncol Biol Phys 1995; 31:113-8.

8. Bel A, Vos PH, Rodrigus PT, Creutzberg CL, et al. High-precision prostate cancer irradiation by clinical application of an offline patient setup verification procedure, using portal imaging. Int $J \mathrm{Ra}$ diat Oncol Biol Phys 1996; 35:321-32.

9. Dawson LA, Mah K, Franssen E, et al. Target position variability throughout prostate radiotherapy. Int J Radiat Oncol Biol Phys 1998; 42:1155-61.

10. Van Lin EN, Nijenhuis E, Huizenga H, et al. Effectiveness of couch height-based patient set-up and an off-line correction protocol in prostate cancer radiotherapy. Int I Radiat Oncol Biol Phys 2001; 50:569-77.

11. Vigneault E, Pouliot J, Laverdiere J, et al. Electronic portal imaging device detection of radioopaque markers for the evaluation of prostate position during megavoltage irradiation: a clinical study. Int J Radiat Oncol Biol Phys 1997; 37:205-12.
12. Hashimoto $S$, Shirato $H$, Nishioka $T$, et al. Remote verification in radiotherapy using digitally reconstructed radiography (DRR) and portal images: a pilot study. Int J Radiat Oncol Biol Phys 2001; 50: 579-85.

13. Hurkmans CW, Remeijer P, Lebesque JV, et al. Set-up verification using portal imaging; review of current clinical practice. Radiother Oncol 2001; 58:105-20.

14. Murphy MJ, Balter J, Balter S, et al. The management of imaging dose during image-guided radiotherapy: report of the AAPM Task Group 75. Med Phys 2007; 34:4041-60.

15. Schallenkamp JM, Herman MG, Kruse JJ, Pisansky TM. Prostate position relative to pelvic bony anatomy based on intraprostatic gold markers and electronic portal imaging. Int $J$ Radiat Oncol Biol Phys 2005; 63: 800-11.

16. Nederveen AJ, van der Heide UA, Dehnad H, et al. Measurements and clinical consequences of prostate motion during a radiotherapy fraction. Int J Radiat Oncol BiolPhys 2002; 53:206-14.

17. Poggi MM, Gant DA, Sewchand W, eta 1. Marker seed migration in prostate localization. Int J Radiat Oncol Biol Phys 2003; 56:1248-51. 